and return in the opposite direction. The only thing that stops them is another $5 / 7$ blemish. If two of the blemishes run headlong into one other, they cancel each other out and disappear.

\section{Light-Emitting Nanofibers Operate as Subwavelength and Multiple Point Sources}

Light-emitting sources with constrained dimensions may play an important role in sensing and lab-on-a-chip applications operating without external optics, enhancing the sensitivity and improving the signal-to-noise ratio of detected fluorescence. J.M. MoranMirabal and co-workers from Cornell University produced point illumination sources based on fibers of $\left[\mathrm{Ru}(\mathrm{bpy})_{3}\right]^{2+}$ $\left(\mathrm{PF}_{6-}\right)^{2} / \mathrm{PEO}$, with electroluminescent ionic transition metal complexes (iTMCs) embedded in a polymer electrolyte on interdigitated electrodes, as reported in the February 14 issue of Nano Letters (p. 458, DOI: $10.1021 /$ nl062778+).

To a solution of $50 \mathrm{mM}$ ruthenium(II) tris(bipyridine) in dry acetonitrile filtered through a $450 \mathrm{~nm}$ polycarbonate membrane, the researchers added poly(ethylene oxide) (PEO) as the carrier polymer, whose concentration tuned the viscosity of the electrospinning solution and affected the size of the fibers with diameters ranging from $150 \mathrm{~nm}$ to several microns. Fibers were electrospun at 8-10 kV on silicon substrates with a 300-600 nm thermal oxide insulating layer and with micropatterned gold interdigitated electrodes (IDEs) on top, using a microfabricated tip coated with gold and keeping the tip-to-substrate distance between $25 \mathrm{~mm}$ and $40 \mathrm{~mm}$.

Fibers deposited on a device having a $5 \mu \mathrm{m}$ interelectrode spacing emitted light confined to a planar region $540 \times 540 \mathrm{~nm}^{2}$ with the out-of-plane dimension limited by the thickness of the fiber when applying a dc bias across the IDEs in a dry nitrogen atmosphere. These devices showed additional emission zones as the voltage increased, resulting in multiple light sources within a fiber. This represents an advantage to develop multiple light sources in parallel, with emission sites defined by interelectrode gaps. Fibers deposited on a device with $500 \mathrm{~nm}$ interelectrode spacing showed a single homogeneous emission zone confined to planar regions of $240 \times 325 \mathrm{~nm}^{2}$ or smaller with the emission spectrum centered at $600 \mathrm{~nm}$, which implies that these devices operate as subwavelength point sources, as well as a reduced turn-on voltage. For the electroluminescent fibers deposited on $5 \mu \mathrm{m}$ IDEs, emission could be detected by a CCD camera when applied voltages were $\sim 10 \mathrm{~V}$ and by eye in a dark room at $100 \mathrm{~V}$. For the electroluminescent fibers deposited on $500 \mathrm{~nm}$ IDEs, emission could be detected at voltages as low as $2.6 \mathrm{~V}$. Relatively long lifetimes were achieved during continuous operation at high voltages, which could be improved, in air, by encapsulation of the light-emitting fibers or substitution of the carrier polymer.

By using other ionic transition metal complexes with emission at different wavelengths in the visible spectrum, nanoscopic light-emitting sources can be produced that excite multiple fluorescent tags, enabling the full integration of excitation and detection mechanisms on lab-ona-chip devices.

JOAN J. CARVAJAL

\section{Integrated Optical Device Fabricated from $\mathrm{KY}\left(\mathrm{WO}_{4}\right)_{2}$}

In the March 1 issue of Optics Letters (p. 488), researchers from the Advanced Photonics Laboratory at the Ecole Polytechnique Fédérale de Lausanne (EPFL, Switzerland), led by M. Pollnau, have reported the fabrication of riblike waveguide structures based on $\mathrm{KY}\left(\mathrm{WO}_{4}\right)_{2}$ (i.e, KYW) doped with $\mathrm{Yb}(\mathrm{KYW}: \mathrm{Yb})$, and codoped with $\mathrm{Lu}^{3+}$ and $\mathrm{Gd}^{3+}$. The researchers also demonstrated the fabrication of a Y-junction with a splitting ratio close to 1:1. According to the researchers, "these riblike structures offer great potential for applications toward integrated optics on KYW."

The researchers said that bulk KYW crystals doped with rare-earth ions are one of the most promising materials for obtaining novel solid-state lasers. In this crystal, the $3+$ rare-earth ions can easily be incorporated, replacing the $\mathrm{Y}^{3+}$ ions. This results in an optically active material with high emission and absorption crosssections for the rare-earth ions. Doping with $\mathrm{Yb}$ ions is of particular interest because an extremely small laser quantum defect $(\sim 1.6 \%)$ can be achieved in $\mathrm{KYb}\left(\mathrm{WO}_{4}\right)_{2}$. The realization of waveguide structures is also of great interest for the development of integrated optical devices.

Recently, in previous work, planar $\mathrm{Yb}$ doped KWY was demonstrated based on high-quality monoclinic KYW:Yb epitaxial layers grown on KYW substrates. In this case, the refractive index contrast $(\Delta n)$ between the active layer and the substrate was only $\sim 6 \times 10^{-4}$. This $\Delta n$ is enough to demonstrate waveguide laser action but not for the development of efficient planar integrated structures with high optical confinement.

In the new work reported here, the researchers grew crack-free KYW:Yb epitaxial layers codoped with $\mathrm{Lu}^{3+}$ and $\mathrm{Gd}^{3+}$ ions. The $\mathrm{Lu}^{3+}$ and $\mathrm{Gd}^{3+}$ ions were optically inert and their role was to increase the refractive index of the active layer. The refractive index contrasts between KYW and $\mathrm{KLu}\left(\mathrm{WO}_{4}\right)_{2}$ and $\mathrm{KLu}\left(\mathrm{WO}_{4}\right)_{2}$ are $\sim 2 \times 10^{-2}$ and $\sim 1 \times 10^{-2}$, respectively. The incorporation of different rare-earth ions in the KYW matrix leads to changes in the lattice parameter. While $\mathrm{Gd}^{3+}$ ions lead to compressive strains, the incorporation of $\mathrm{Yb}^{3+}$ and $\mathrm{Lu}^{3+}$ ions induces tensile strains. Therefore, by adjusting the concentration of the different rare-earth ions, it is possible to achieve an epitaxial layer characterized by a high refractive index and a low lattice-induced strain, and with large flexibility concerning the concentration of the optically active $\mathrm{Yb}^{3+}$ ions.

The epitaxial Lu,Gd-codoped KYW:Yb layers were grown by liquid phase epitaxy. Using concentrations of $25.3 \%$ for the $\mathrm{Lu}$, $13 \%$ for the $\mathrm{Gd}$, and 1.7 at \% for $\mathrm{Yb}$, the researchers were able to grow crack-free layers with a thickness of up to 8-10 $\mu \mathrm{m}$ and with a $\Delta n=7.5 \times 10^{-3}$. Subsequently, two-dimensional waveguide structures were fabricated using standard UV photolithography and reactive ion etching. Ribs with widths from $3 \mu \mathrm{m}$ to $9 \mu \mathrm{m}$ exhibit single mode behavior. The researchers established that the losses at the fluorescent wavelength of $1020 \mathrm{~nm}$ will be below $1 \mathrm{~dB} / \mathrm{cm}$ for straight waveguides. In order to analyze the potential for building integrated optical devices, the researchers have designed and produced a Y-splitter with a splitting ratio of 1:1 and additional losses of $1.4 \mathrm{~dB}$.

Rosalía SERna

\section{Nanoelectrodes Probe Microscale Environments}

Investigating the composition and behavior of microscale environments, including those within living cells, could become easier and more precise with nanoelectrodes being developed at the University of Illinois at UrbanaChampaign (UIUC).

"The individual nanotube-based probes can be used for electrochemical and biochemical sensing," said M.-F. Yu, a professor of mechanical science and engineering at UIUC, and a researcher at the university's Beckman Institute. "The position of the nanoelectrodes can be controlled very accurately."

To fabricate the nanoelectrodes, $\mathrm{Yu}$ and graduate students K. Yum, J. Hu, and H.N. Cho begin by attaching a strong, rigid, boron-nitride nanotube to a much larger, conductive probe. The nanotube will form the insulating core of the nanoelectrode.

The researchers then coat the nanotube with a thin film of gold $\sim 10-50$ nm thick. 